\title{
Clinical evaluation of dimethyl fumarate for the treatment of relapsing-remitting multiple sclerosis: efficacy, safety, patient experience and adherence
}

This article was published in the following Dove Press journal: Patient Preference and Adherence

\author{
Bhavya Narapureddy' \\ Divyanshu Dubey ${ }^{1,2}$
}

'Departments of Neurology Mayo Clinic, Rochester, MN, USA; ${ }^{2}$ Laboratory Medicine and Pathology Mayo Clinic, Rochester, MN, USA
Correspondence: Divyanshu Dubey Department of Laboratory Medicine \& Pathology, and Neurology, 200 First Street S.W., Rochester, MN 55905, USA Email Dubey.divyanshu@mayo.edu

\begin{abstract}
Dimethyl fumarate (DMF) is an oral disease-modifying therapy approved for management of relapsing-remitting multiple sclerosis patients. Results from phase 3 clinical trials (DEFINE, CONFIRM) and follow-up study (ENDORSE) have provided good evidence for its efficacy and safety profile. Patient-reported outcomes (PROs) assessment revealed stabilization or boost in health-related quality of life and work productivity of patients treated with DMF compared to placebo reflecting a higher patient satisfaction to therapy. Being an oral agent with relatively favorable risk versus benefit profile DMF is commonly prescribed first-line agent. However, literature suggests that intolerance to side effects, especially gastrointestinal adverse effects and flushing is one of the major causes to compromised therapeutic compliance. An increase in the real-world incidence of progressive multifocal leukoencephalopathy and liver abnormality cases is also concerning. Several prevention and mitigation strategies like patient counseling, dose up-titration, pretreatment with aspirin, use of symptomatic therapy and frequent blood monitoring have demonstrated to be effective in tackling these adverse effects and promoting adherence to DMF. In this article, we review the efficacy, safety, PROs and patient adhere data, along with various measures to manage adverse events and promote compliance.
\end{abstract}

Keywords: dimethyl fumarate, multiple sclerosis, drug safety, patient adherence

\section{Introduction}

Multiple sclerosis (MS) is a chronic inflammatory demyelinating disorder of the central nervous system characterized by dysregulation of both innate and adaptive immune responses. ${ }^{1}$ Though the pathogenesis is unclear, interplay between genetic, lifestyle and environmental factors play a major role. ${ }^{2}$ The incidence of MS has escalated over the past few decades and the disorder typically afflicts young adults with a higher incidence in women. ${ }^{3}$ Relapsing-remitting multiple sclerosis (RRMS) is the most common initial presenting subtype, characterized by distinct relapses alternating with a period of either full recovery or incomplete recovery. ${ }^{4}$ Seventeen disease-modifying therapies (DMTs) are currently available in the market for treatment of relapsing-remitting multiple sclerosis (RRMS). ${ }^{5-7}$ These include oral, self-injectable and infusible medications with varied mechanisms of action and degrees of efficacy. ${ }^{8}$ Due to fear of needles, difficulty with self-injection, injection-site reactions and a range of infusion-associated reactions like headache, rash, pyrexia, nausea and flushing, oral forms of therapy may appear to be more appealing to the patients. ${ }^{8-10}$ Prior studies have also demonstrated greater adherence and persistence to oral DMTs compared with injectable DMTs. ${ }^{11,12}$ 
Dimethyl fumarate (DMF) was approved by the US Food and Drug Administration and European Medicines Agency during March 2013 for relapsing forms of MS. Though recently approved for relapsing forms of MS, DMF use for psoriasis dates back to 1990s and has widely published favorable efficacy and safety data in the literature. ${ }^{13}$ DMF is one of the first-line agents for treatment of new-onset RRMS with intermediate disease activity. ${ }^{14}$ Due to ease of administration, favorable efficacy and adverse effect profile, DMF was one of the most prescribed oral medication post approval. ${ }^{3}$ However, with more than 5 years of real-world use, it is crucial to evaluate patient's experiences and acceptance to the treatment.

\section{Mechanism of action}

The exact mechanism of action of DMF has not yet been fully elucidated. Most studies conducted so far propose that the therapeutic benefits of DMF are primarily through immunomodulatory and antioxidative mechanisms.

\section{Antioxidative effect}

Due to its ability to covalently modify proteins, a rapid decline in major intracellular antioxidant Glutathione (GSH) is seen following exposure to fumaric acid esters. ${ }^{15}$ Decreased levels of GSH lead to an upregulation in transcription of nuclear factor erythroid 2-related factor 2 (Nrf2). Furthermore, monomethyl fumarate (MMF), which is an active metabolite of DMF, causes modification of cysteine residue 151 of Kelch-like ECH-associated protein 1 (KEAP1) which normally induces ubiquitinmediated degradation of Nrf2. Such modification prevents the interaction between the two molecules and stabilizes Nrf2. ${ }^{16}$ The resultant increase in Nrf2 proteins translocate to the nucleus and activate transcription of several antioxidant genes like glutathione S-transferases (GST), heme oxygenase 1 (HO-1) and $\mathrm{NAD}(\mathrm{P}) \mathrm{H}$ : quinone oxidoreductase (NQO1) and subsequent increase in the production of GSH. ${ }^{17}$ DMF has also been shown to prevent oxidative stress-induced apoptosis and promote survival of neural stem/progenitor cells and differentiated neurons by inducing Nrf2 ERK1/2-MAPK pathway. ${ }^{18}$ This is supported by the termination of Nrf2 accumulation upon administering ERK1/2 inhibitor, PD98059. ${ }^{18}$

\section{Immunomodulatory effects}

DMF and its active metabolite MMF exert a variety of immunomodulatory effects primarily through impairment of NF-kB signaling. Depletion of intracellular GSH stores by DMF upregulates heme oxygenase-1 (HO-1) production, which binds to NF- $\mathrm{KB}$ and prevents the transcription of IL-23p19 gene. DMF also impairs STAT1 phosphorylation leading to inhibition of IL-12p35 transcription. This causes type II dendritic cells to produce more interleukin-10 (IL-10) instead of IL-12 or IL-23. HO-1 also inhibits T cell proliferation by decreasing MHC class II expression on antigen-presenting cells. ${ }^{19}$ Furthermore, DMF inhibits a nuclear kinase, MSK1 mediated phosphorylation of NF- $\mathrm{KB} / \mathrm{P} 65$ subunit and histone-3 at serine 10 of DNA leading to suppression of P65 ability to bind to DNA and thereby impede its transcriptional activity. ${ }^{20} \mathrm{DMF}$ is also shown to inhibit TNF alpha and IL-10 induced nuclear accumulation of NF-kB/P50 subunit ${ }^{21}$ and prevents I $\mathrm{I} B \alpha$ degradation, which normally binds to and sequesters NF- $\mathrm{KB}$ proteins in the cytoplasm. ${ }^{22}$ Ultimately, suppressing NF- $\mathrm{\kappa B}$ signaling was found to reduce nitric oxide synthetase 2 (NOS2), IL-6 and IL-12 gene expression, thereby preventing pro-inflammatory $\mathrm{T}$ helper cell differentiation and also decreasing nitric oxide-induced oligodendrocyte and neuronal damage..$^{20,22}$

DMF induces apoptosis of $\mathrm{T}$ cells by decreasing expression of the anti-apoptotic protein $\mathrm{Bcl}-2$ and also due to upregulation of Apo2.7, which leads to DNA fragmentation. ${ }^{23}$ In addition, DMF exhibits immunomodulation by biasing toward CCR3+Th2 subsets of $\mathrm{T}$ cells. This leads to an increase in anti-inflammatory cytokines, IL-4 and a decrease in pro-inflammatory cytokines, IFN- $\gamma$ and IL-17. ${ }^{24}$ DMF has also shown to alter regulatory T cell population by specifically enhancing pTreg subsets, which are known to promote peripheral tolerance. ${ }^{25}$

Activation of hydroxycarboxylic acid receptor 2 (HCAR2) by MMF in experimental autoimmune encephalomyelitis (EAE) mice led to a shift in molecular and functional phenotypes of activated microglia from proinflammatory to neuroprotective type. Also, HCAR2mediated activation of downstream AMPK-Sirt1 axis led to inhibition of NF- $\mathrm{kB}$. DMF restored synaptic alterations by modulating glutamate release in the corticostriatal tracts of the mice. ${ }^{26}$ It also reduced demyelination of spinal cord and immune cell infiltration through interfering neutrophil adhesion to endothelial cells and chemotaxis in wild type mice. However, this effect was not observed in HCA2-/- mice indicating the potential role of HCA2 in DMF-induced neuroprotection. ${ }^{27}$

\section{Pharmacokinetics}

DMF is administered orally in the form of enteric-coated delayed-release capsules to prevent fumaric acid-induced 
gastric irritation. ${ }^{28}$ It is almost completely and rapidly hydrolyzed by esterases to an active metabolite MMF in the alkaline milieu in small intestine mucosa. Remaining DMF is hydrolyzed either in the portal vein/plasma or forms a GSH adduct which is metabolized to mercapturic acid and is later excreted in the urine. Only MMF was detected in the plasma indicating its predominance in biological effects in vivo. MMF is further metabolized to form $\mathrm{H}_{2} \mathrm{O}$ and carbon dioxide through tricarboxylic acid cycle. DMF does not involve in any potential drug to drug interactions as no CYP metabolism was identified. ${ }^{28,29}$ The mean time taken for MMF to reach peak concentration ( $\mathrm{T}$ max) following administration of $240 \mathrm{mg}$ DMF twice daily (BID) and thrice daily (TID) was 4 and $6 \mathrm{hrs,}$ respectively. The average half-life of MMF was observed to be $0.81 \mathrm{hrs}$ (BID) and $0.85 \mathrm{hrs}$ (TID). No accumulation of MMF was observed with multiple dosing of DMF. ${ }^{30}$ The relatively short half-life and lack of accumulation highlight the importance of medication compliance. Coadministration of DMF with high calorie and fat-rich food is shown to delay $\mathrm{T}$ max for up to a few hours and a decrease in the peak plasma concentration ( $\mathrm{C} \max$ ) by $40 \%$; however, it does not modify the AUC. This delayed metabolism has shown to decrease side effects and increase patient tolerability. ${ }^{31} \mathrm{~A}$ recent study analyzing CSF samples following administration of oral DMF showed that peak plasma concentration (C max) of MMF in the CSF ranged in between 39 and $79 \mathrm{ng} / \mathrm{mL}$ and $\mathrm{T}$ max was $7 \mathrm{hrs}$. Interestingly, the ratio of CSF to plasma MMF concentration post $7 \mathrm{hrs}$ was $15 \%$, indicating that MMF can potentially cross the blood-brain barrier and exert a direct neuroprotective effect. ${ }^{32}$

\section{Efficacy \\ Clinical efficacy}

Two phase 3 studies, namely CONFIRM and DEFINE have demonstrated efficacy of DMF in the management of RRMS. In the DEFINE study, there was a significant reduction in annualized relapse rate (ARR) in DMF group (53\% with BID and $48 \%$ with TID) as compared to placebo at 2 years. ${ }^{33}$ A significant reduction in proportions of patients with confirmed progression of disease at 3 months (38\% with BID and $34 \%$ with TID) was noted. ${ }^{33}$ In the CONFIRM study, DMF-associated reduction in ARR was $44 \%$ (in BID dosing) and 51\% (in TID dosing) over a period of 2 years compared to placebo. ${ }^{34}$ Interestingly, DMF caused rapid (ie, within 12 weeks of initiation of
DMF) and sustained reduction in ARR and cumulative risk of relapse compared to placebo. ${ }^{35}$ In the CONFIRM study, DMF was associated with a notable decrease in disability progression compared to placebo, but the difference was not statistically significant. However, an integrated analysis of both the DEFINE and CONFIRM studies demonstrated a significant reduction in confirmed disability progression measured by Expanded Disability Status Scale (EDSS) both at 12-weeks and 24 weeks compared to placebo. ${ }^{36}$ Integrated post-hoc analysis also showed a significant association of DMF with functional improvements measured by Multiple Sclerosis Functional Composite (MSFC) score compared to placebo (mean change in MSFC was 0.054 in DMF vs -0.053 in placebo). ${ }^{37}$

DMF has demonstrated a remarkable neuroradiological efficacy. Results from the MRI cohort of DEFINE and CONFIRM study showed a significant reduction in number of new or enlarging T2 hyperintense and Gadoliniumenhancing lesions at 6 months and the effect was sustained at 1 and 2 years. ${ }^{34,38}$ A small retrospective study conducted in a real-world setting correspondingly showed a lower rate of whole-brain atrophy over a period of 1 year. ${ }^{39}$ Additionally, whole-brain magnetization transfer ratio (MTR), a likely measure for assessing myelin density in brain tissue analyzed from the DEFINE study showed a significant increase in myelin density of $0.129 \%$ with DMF BID and $0.096 \%$ with DMF TID over 2 years whereas decrease in myelin density was noticed in placebo group $(-0.386 \%)$, substantiating neuroprotective role of DMF. ${ }^{38}$

No evidence of disease activity (NEDA) status is a helpful hybrid measure used in validating therapeutic response. NEDA status is considered to be achieved if there are no relapses, no 12-week disability progression (clinical NEDA) and no MRI activity (neuro radiological NEDA). Integrated analysis of the MRI cohort of DEFINE and CONFIRM trials showed that a significantly higher percentage of patients achieved overall NEDA in the DMF treatment group compared to placebo over 2 years ( $26 \%$ vs $12 \%$ ), with a relative risk reduction of $42.7 \%{ }^{40}$

Sustained clinical and neuroradiological efficacy have been demonstrated in long-term extension of these studies (ENDORSE). ${ }^{41}$ Five-year interim results (2 years of CONFIRM/DEFINE and 3 years of ENDORSE) showed low clinical and MRI disease activity and acceptable safety profile for patients continuing DMF BID or TID dosing. Several post-marketing and real-world studies 
published so far have substantiated the long-term efficacy of DMF. ${ }^{42-45}$

\section{Patient-reported outcomes}

Assessing patient experiences by means of patientreported outcomes (PROs) is valuable in substantiating the effectiveness of treatment. ${ }^{46} \mathrm{MS}$ is known to significantly impact the quality of life of patients. ${ }^{47}$ Healthrelated quality of life (HRQoL) assessment was conducted at baseline and approximately every 12-24 weeks in both the phase 3 studies using 3 PROs, namely, Short form (SF36 questionnaire), EuroQol-5D (EQ-5D) instruments and patient global assessment of well-being visual analog scale (VAS). Greater impairment in HRQoL was noticed in patients with higher disability scores and those who had a relapse. DMF was found to either significantly increase or stabilize HRQoL scores of treated patients whereas a decline was noticed in the placebo group. In patients who had $\geq 1$ relapse during the study period, treatment with DMF was shown to reduce the impact of relapse as evident by lesser extent of reduction or even improvement in SF36 Physical component scores (PCS) and Mental component scores (MCS) as compared to steady decline in the placebo group (Table 1). ${ }^{48,49}$ Such improved patient-perceived health status following treatment with DMF can positively influence patient acceptance and promote treatment satisfaction. Convincingly, two large observational studies conducted, namely, PROTEC and ESTEEM study showed a stable or higher PROs scores with the use of DMF in the real world similar to the effect seen in phase 3 trials. $^{42,43}$ It is worth noting that patients treated with DMF had a more positive impact on HQRoL and subsequently work productivity compared to other first-line therapies like interferon and glatiramer acetate. The data collected using patient self-completion forms showed higher HRQoL and work productivity outcomes in DMF patients than in patients receiving an interferon or glatiramer acetate therapy. 50

\section{Side effects}

DMF has demonstrated a good safety and tolerability profile in two major phase 3 clinical trials. Flushing and gastrointestinal (GI) events (which included diarrhea, upper abdominal pain, nausea, vomiting) were the common symptoms patients experienced after taking DMF. In an integrated analysis of CONFIRM/DEFINE, the incidence of GI adverse events (AEs) was 40\% in DMF group as compared to $31 \%$ in the placebo group over 2 years. Similarly, the incidence of flushing was $45 \%$ in the DMF group compared to $8 \%$ in the placebo group. Notably, the incidence of flushing and GI AEs was highest in the first month and thereafter reduced significantly. ${ }^{51}$ The incidence of serious adverse effects was similar across both groups. No incidence of malignancies was reported. The overall incidence of infections across all the treatment groups was similar. Most common infections reported in the DMF group included nasopharyngitis, urinary tract infections, upper respiratory tract infection, bronchitis and influenza. $^{33,34}$

Long-term follow-up data obtained from the ENDORSE study also demonstrated a favorable risk-benefit profile of DMF. Patients who continued on DMF more commonly reported multiple sclerosis relapse and nasopharyngitis whereas flushing and GI events were more common in patients new to DMF. However, the overall incidence of adverse events including serious adverse events was similar across all the treatment groups. ${ }^{41}$

DMF is known to cause apoptosis of lymphocytes resulting in lymphocytopenia. In DEFINE/CONFIRM, the lymphocyte count was reported to decrease by approximately $30 \%$ during the 1 st year and later the levels plateaued. Around $6 \%$ of the patients developed grade 3 lymphopenia (absolute lymphocyte count (ALC) of less than $0.5 \times 10^{9} / \mathrm{L}$ ) in the treatment group vs less than $1 \%$ in the placebo group. ${ }^{33,34}$ Five-year interim analysis of the ENDORSE study revealed that the incidence of grade 3 lymphopenia was $7-8 \%$ in patients continuing DMF during extension phase and $6-9 \%$ in patients who were switched from placebo to $\mathrm{DMF}^{41}$ In a recent real-world study of 405 patients on DMF therapy, the incidence of lymphopenia was higher than that observed in phase 3 studies $(17 \%$ of the patients developed grade 2 lymphopenia $\left[<800 / \mathrm{mm}^{3}\right]$ and $11 \%$ had grade $2+3$ lymphopenia $\left.\left[<500 / \mathrm{mm}^{3}\right]\right){ }^{52}$ The risk of lymphopenia was found to increase with older age especially $>55$ years, patients with low baseline ALCs, longer disease duration and those previously on natalizumab. ${ }^{44,45}$ Patients with grade 2 or above lymphopenia had persistently low counts often necessitating cessation of treatment and were observed to normalize only after a period of $>5$ months. ${ }^{45}$

Nineteen cases of Progressive multifocal leukoencephalopathy (PML) have been reported following use of fumaric acid esters, of which 5 were reported in RRMS patients. Among those 5 patients, 3 had persistent grade 3 lymphopenia for $>6$ months and 1 had grade 2 lymphopenia. ${ }^{53}$ However, all the 19 cases had low CD4+ 
Table I Summary of patient-reported outcomes from phase 3 trials

\begin{tabular}{|c|c|c|c|c|c|c|c|c|}
\hline \multirow[t]{3}{*}{ Study } & \multicolumn{4}{|l|}{ DEFINE $^{49}$} & \multicolumn{4}{|l|}{ CONFIRM $^{48}$} \\
\hline & \multirow[t]{2}{*}{ Placebo } & \multicolumn{3}{|c|}{ BG I 2 (240 mg) } & \multirow[t]{2}{*}{ Placebo } & \multicolumn{3}{|c|}{ BG I 2 (240 mg) } \\
\hline & & \multicolumn{2}{|c|}{ BID } & TID & & \multicolumn{2}{|c|}{ BID } & TID \\
\hline \multicolumn{9}{|l|}{ SF- 36} \\
\hline Evaluable patients $(n)$ & 389 & \multicolumn{2}{|l|}{400} & 400 & 344 & \multicolumn{2}{|c|}{341} & 328 \\
\hline PCS scale at baseline (Mean, SD) & $43.3(10.2)$ & \multicolumn{2}{|c|}{$42.9(10.02)$} & $43.7(10.80)$ & $42.94(9.90)$ & \multicolumn{2}{|c|}{$43.07(9.91)$} & $43.02(10.0)$ \\
\hline Improved (\%) & 16.2 & \multicolumn{2}{|l|}{21.8} & 24.0 & 19.2 & \multicolumn{2}{|l|}{22} & 26.2 \\
\hline Stable (\%) & 56.8 & \multicolumn{2}{|l|}{59.8} & 58.8 & 57 & \multicolumn{2}{|c|}{58.7} & 52.7 \\
\hline Worsened (\%) & 27.0 & \multicolumn{2}{|l|}{18.5} & 17.3 & 23.8 & \multicolumn{2}{|c|}{19.4} & 21 \\
\hline OR for improvement & & \multicolumn{2}{|l|}{1.47} & 1.79 & & \multicolumn{2}{|c|}{1.22} & 1.6 \\
\hline$P$-value & & \multicolumn{2}{|c|}{0.0498} & 0.0027 & & \multicolumn{2}{|c|}{0.3186} & 0.0188 \\
\hline MCS scale at baseline (mean, SD) & 45.7 (11.15) & \multicolumn{2}{|c|}{$45.3(10.93)$} & $45.1(10.68)$ & $44.8(10.73)$ & \multicolumn{2}{|c|}{$45.4(11.7)$} & $44.9(10.75)$ \\
\hline Improved, (\%) & 20.6 & \multicolumn{2}{|l|}{25.8} & 30 & 25.9 & \multicolumn{2}{|c|}{27.6} & 30.2 \\
\hline Stable (\%) & 50.1 & \multicolumn{2}{|l|}{50.5} & 49.8 & 45.3 & \multicolumn{2}{|c|}{44.6} & 43.3 \\
\hline Worsened (\%) & 29.3 & 23.8 & & 20.3 & 28.8 & 27.9 & & 26.5 \\
\hline OR for improvement & & 1.44 & & 1.93 & & 1.20 & & 1.39 \\
\hline$P$-value & & 0.065 & & 0.0008 & & 0.36 & & 0.1024 \\
\hline & With relapse & & With & ut relapse $(n=948)$ & With relapse & 54) & With & ut relapse $(n=835)$ \\
\hline PCS score (mean, SD) & & & & & & & & \\
\hline Change from baseline to 2 years & $-2.10(7.45)$ & & 0.72 & 6.94) & $-0.97(8.07)$ & & 0.57 & 7.38) \\
\hline$P$-value & $<0.0001$ & & & & $<0.0001$ & & & \\
\hline MCS score (mean, SD) & & & & & & & & \\
\hline Change from baseline to 2 years & $-0.47(10.05)$ & & 0.31 & $9.25)$ & $-0.67(10.58)$ & & 0.52 & $9.71)$ \\
\hline$P$-value & 0.6635 & & & & 0.0112 & & & \\
\hline EQ-5D index score (mean, SD) & & & & & & & & \\
\hline Change from baseline to 2 years & $-0.02(0.20)$ & & 0.01 & $0.01)$ & $-0.01(0.22)$ & & 0.01 & $0.19)$ \\
\hline$P$-value & 0.0004 & & & & 0.0005 & & & \\
\hline EQ-5D VAS (mean, SD) & & & & & & & & \\
\hline Change from baseline to 2 years & $-6.03(17.86)$ & & 0.52 & | 5.99) & $-4.04(19.20)$ & & 0.30 & 17.52) \\
\hline$P$-value & $<0.0001$ & & & & $<0.0001$ & & & \\
\hline
\end{tabular}

Abbreviations: SF-36, Short Form 36; PCS, Physical Component Summary (physical functioning, role-physical, bodily pain and general health); MCS, Mental Component Summary (vitality, social functioning, role-emotional and mental health); EQ-5D, mobility, self-care, usual activities, pain/discomfort and anxiety/depression; SD, standard deviation; TID, thrice daily; BID, twice daily; OR, odds ratio.

and CD8 $+\mathrm{T}$ cells, with a more pronounced reduction in CD8+ T cells. ${ }^{54}$ A recently published case of a 76-year-old patient on DMF treatment (since April 2014) was incidentally found to have John Cunningham virus (JCV) DNA in the CSF $(1,988,880$ copies $/ \mathrm{mL})$ and serum and highly positive anti-JCV-antibody in CSF and serum despite no symptoms of PML or global lymphopenia (ALCs were always $>1240$ ). However, there was a reduction in CD8+ 
$\mathrm{T}$ cell levels and an increase in $\mathrm{CD} 4+/ \mathrm{CD} 8+$ ratio despite normal ALCs suggesting the importance of monitoring these subsets. ${ }^{55}$

Hepatic dysfunction or transaminitis is another potential adverse effect of DMF. A higher percentage of patients with elevations of liver aminotransferase levels more than 3 times upper the normal limit were observed in DMF treated group compared to placebo in the DEFINE study (6\% vs 3\%) whereas no difference across study groups was noticed in the CONFIRM study. ${ }^{33,34}$ Although no cases of hepatic failure have been reported in phase 3 trials, a total of 14 clinically significant liver injury cases were observed following real-world use of DMF either due to DMF hypersensitivity, infection or autoimmune hepatitis, with onset as early as a month of initiation. ${ }^{56}$

\section{Patient adherence information}

Multiple sclerosis patients are subjected to long-term treatment regimens and thus, adherence to medication plays a crucial role in disease control. Higher treatment adherence also decreases the economic burden on patients by decreasing MS-related hospitalizations, relapses and related costs. $^{57}$

In the DEFINE study, the discontinuation rates across DMF and placebo groups were similar (31\% and 35\%, respectively). Specifically, the proportion of patients who switched to other MS medications was lower in DMF group (6\% in BID and 5\% in TID BG 12 group) as compared to placebo $(13 \%){ }^{33}$ In the CONFIRM study, the rate of discontinuation and the proportion of switch to alternative MS medications was higher in the placebo group as compared to the DMF-treated group (36\% vs $30 \%$ in BID, $28 \%$ in TID group). ${ }^{34}$ The overall discontinuation rates secondary to adverse effects in both the studies were comparable between treatment and placebo group with flushing and GI AEs more significantly contributing to discontinuation (Figure 1). ${ }^{51}$ ENDORSE data showed that the patients new to DMF therapy had higher rates of discontinuation secondary to adverse events (PBO to $\mathrm{DMF}-14-26 \%$ ) as compared to those who were

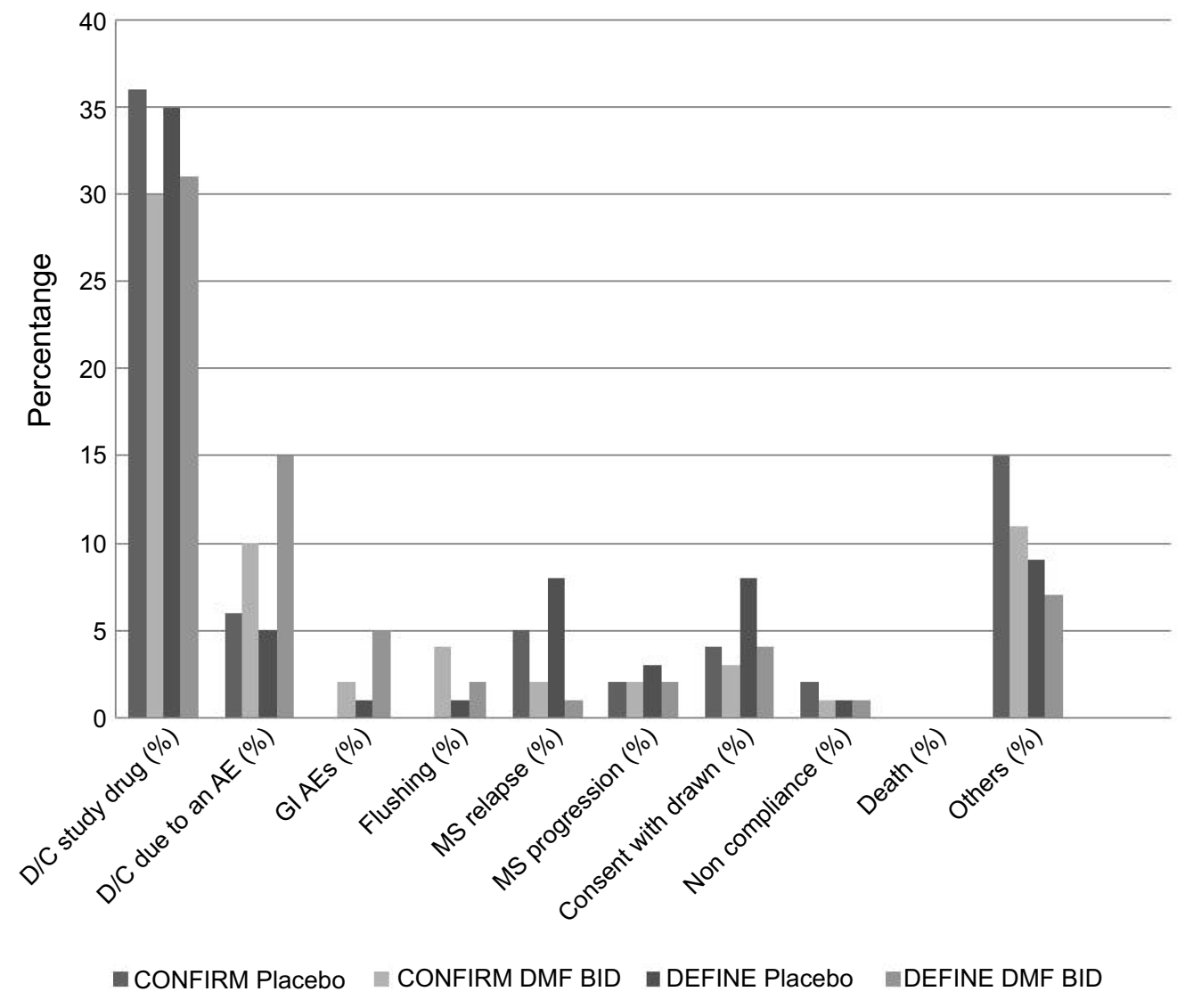

Figure I Dimethyl fumarate (DMF) patient adherence data from phase 3 studies.

Abbreviations: D/C, discontinued; BID, twice daily; MS, multiple sclerosis; Gl, gastrointestinal; AE, adverse effects; Others, personal reasons or decisions, moving to another geographic area, desire to become pregnant, actual pregnancy, lost to follow-up, investigator decision, perceived lack of efficacy and having previously met the protocol-defined relapse criteria for alternative MS medication. 
continued on DMF treatment $(6-7 \%) .{ }^{41}$ This significant difference in discontinuation rates is largely due to GI disturbance and flushing occurring early in DMF therapy.

Several post-marketing studies have also evaluated tolerability and adherence to DMF (Table 2). An open-label, single-arm study (TOLERATE) was conducted to assess GI tolerability in the real world using eDiaries to document GI events (frequency, severity, duration) and also mitigation strategies for a period of 12 weeks. ${ }^{58}$ The study showed that $14.7 \%$ of the patients on DMF discontinued treatment, of which $10 \%$ was due to AEs. A total of $6.6 \%$ patients discontinued due to GI AEs despite receiving symptomatic treatment. Also, DMF induced hypersensitivity reaction and elevation of AST, ALT and GGTs were reported to cause treatment discontinuation. In another study (MANAGE), the discontinuation rates due to AEs were found to be $9.9 \%$, of which $7.3 \%$ of the patients dropped out treatment due to GI intolerance. ${ }^{59}$ Furthermore, persistent lymphopenia due to DMF prompted discontinuation of treatment in clinical practice unlike in CONFIRM trial where discontinuation due to this reason was not reported. ${ }^{44}$ Notably, in a population-based cohort study of 400 patients, only $43 \%$ of the patients remained persistent (ie, no treatment gap $\geq 60$ days or switching to another DMT) at the end of 2-year treatment reflecting low persistence to DMF in the real world compared to phase 3 trials. $^{60}$ Although DMF has shown to be equally efficacious as fingolimod and superior to teriflunomide, ${ }^{61}$ several studies published so far comparing the tolerability profile of these oral-administered DMTs suggest that DMF has lower adherence and persistence compared to either fingolimod or teriflunomide especially due to adverse effects, indicating a compelling need to manage intolerability to dimethyl fumarate treatment. ${ }^{62,63}$ The frequency of dosing is also an important factor to effect adherence to the treatment. Even though per oral administration is more convenient, studies have shown the multiple-daily dosing can influence patient's choice and compliance. $^{64,65}$

\section{Measures to promote treatment adherence}

Given the evidence of good clinical and radiological efficacy in pivotal trials and in clinical practice, there is a necessity to address poor adherence and prevent treatment discontinuation.

\section{Patient education and setting expectations}

Patient counseling and expectation setting prior to treatment initiation have been recommended by experts as a very effective means to foster adherence and persistence to therapy. ${ }^{66} \mathrm{~A}$ standard nursing initiation protocol also called the New York University (NYU) initiation protocol developed by a nursing team at NYU MS comprehensive center reflects the importance of effective nursing strategies and education in improving DMF tolerability. ${ }^{67}$ The protocol includes educating the patient and family members prior to treatment initiation, providing them with written and verbal instructions to prevent and manage side effects, encouraging use of weekly diary to assist in

Table 2 Real-world evidence to dimethyl fumarate adherence

\begin{tabular}{|l|l|l|l|}
\hline Study name & Miclea et al, 2016 & Smoot et al, 2017 & Sejbaek et al, 2018 $^{\mathbf{7 5}}$ \\
\hline Type of study & Retrospective study & Prospective study & Retrospective study \\
\hline Number of patients evaluated & 644 & 412 & 253 \\
\hline Sex ratio M/F & $193 / 451$ & $96 / 316$ & $77 / 176$ \\
\hline Treatment naive patients, \% & 45.2 & 30 & 29.2 \\
\hline Discontinuation of DMF, \% & 28.7 & 38 & 27.7 \\
\hline Discontinuation due to AE, \% & 22.2 & 28.6 & 21.3 \\
Gastrointestinal AEs, \% & 12.7 & 15 & 10.9 \\
Lymphopenia, \% & 5.3 & 2.4 & 2.6 \\
Flushing, \% & 1.7 & 5.1 & 3.2 \\
Other AEs, \% & 2.5 & 12.8 & 4.6 \\
\hline Discontinuation due to relapse/inefficacy/MRI worsening, \% & 6.5 & 6.3 \\
\hline
\end{tabular}

Note: Other AEs include rash, elevated ALT, depression, edema, arthralgia.

Abbreviations: DMF, dimethyl fumarate; $M / F$, male/female; $A E$, adverse effects; $M R I$, magnetic resonance imaging. 
tracking side effects and mitigation strategies, reviewing safety monitoring protocols, regular follow-ups to assess medication tolerability. A retrospective study comparing discontinuation rates in patients who followed the NYU protocol versus the Biogen protocol demonstrated higher tolerability and lower rates in discontinuation at 6 weeks with the NYU protocol $(2.5 \%$ vs $12 \%) .{ }^{67}$ In a web-based survey conducted in German MS patients using anonymous questionnaire, it was observed that patients who had realistic expectations of treatment outcomes, reliable information sources and good self-management skills strongly correlated with adherent behavior. Individualized coaching calls offered through patient counseling programs (PCP) to the participants based on their adherent behavior reported to have a low therapy dropout rate $(3.7 \%)$ as compared to those who were not coached signifying the importance of good support system in promoting patient adherence. ${ }^{68}$

\section{Gastrointestinal adverse effects}

Though gastrointestinal adverse effects are transient, they are extremely unpleasant for patients. Through a Delphi process, a consensus was reached among North American clinicians on strategies to manage GI AEs. Based on the survey results, co-administration of DMF with a meal is considered an effective way of minimizing the incidence and severity of GI AEs. High fat (such as peanut butter, yogurt, and cheese), high protein and low starch food is recommended by most of the responders. ${ }^{69}$ In both MANAGE and TOLERATE studies, patients were advised to take the medication along with or within $1 \mathrm{hr}$ of meal. A modest reduction in the occurrence of GI AEs was observed in patients who consistently took DMF with meal. Strikingly, in the MANAGE study, the percentage of patients with severe GI outcomes was significantly lower (7.7\%) in patients who took DMF regularly with food compared to those who did not $(15.5 \%)$. However, only $17 \%$ of the participants in MANAGE and $24 \%$ in TOLERATE complied regularly with these instructions. ${ }^{58,59}$ According to Biogen prescribing information, patients are recommended to take $120 \mathrm{mg}$ DMF BID for first 7 days as a starter dose followed by $240 \mathrm{mg}$ BID as maintenance dose, $^{31}$ but slower titration for more than 7 days ( $\leq 4$ weeks) to maintenance dose was found to increase GI tolerability. ${ }^{69}$ However, a study conducted in healthy volunteers receiving slow titration of DMF showed no effects on GI tolerability. ${ }^{70}$
A modified titration schedule has been proposed by a nursing team as part of NYU initiation protocol recommends dosing as follows: $120 \mathrm{mg}$ OD for first 14 days, followed by $240 \mathrm{mg}$ OD for next 14 days, followed by 240 mg BID. Interestingly, a retrospective chart review showed that patients who followed NYU initiation protocol had higher GI tolerability compared to patients on Biogen protocol $(1.9 \%$ vs $8 \%){ }^{67}$

Temporary dose reduction is another option to promote GI tolerability in patients who develop severe GI AEs while on $240 \mathrm{mg}$ BID. It is advisable to switch to 120 mg BID for 1 to 2 weeks and slowly re-titrate to maintenance dose over a period of 4 weeks. In patients who are unable to tolerate even after re-titration, discontinuation of DMF is considered. ${ }^{31,69}$ Symptomatic management of GI AEs is recommended by experts to alleviate symptoms. In a Delphi study, most clinicians agreed upon using ondansetron, bismuth subsalicylate and promethazine for nausea and vomiting, antacids for nausea, bismuth subsalicylate, antacids and anti-secretory drug treatment for abdominal pain and loperamide and diphenoxylate/atropine for diarrhea. ${ }^{69}$ In both the MANAGE and TOLERATE studies, the severity of GI AEs was reduced with the use of symptomatic management. Notably, use of symptomatic therapy was maximum during first 1-4 weeks, and by 12 th week, only $10 \%$ of the patients in the MANAGE study and $3.3 \%$ of the patients in the TOLERATE study required symptomatic therapy. ${ }^{58,59}$ A recent placebo-controlled study (PREVENT) demonstrated that bismuth subsalicylate is effective in reducing the incidence and severity of flatulence and diarrhea as compared to placebo. $^{71}$ A small single-arm study demonstrated the efficacy of $10 \mathrm{mg}$ montelukast in attenuating GI AEs. ${ }^{72}$ However, in the MITIGATE study, montelukast administration was not associated with any significant change in the incidence and severity of GI AEs as compared to placebo. $^{73}$

\section{Flushing}

Pretreatment with aspirin is proven to be successful in mitigating flushing. A study conducted in healthy volunteers showed that pretreatment with $325 \mathrm{mg}$ of aspirin (ASA) 30 mins before DMF dosing reduced the incidence, severity and the number of flushing events compared to the placebo group, but no effect was noticed on the duration of each event. ${ }^{70}$ Use of aspirin had no adverse impact on GI-related events or on pharmacokinetic parameters or efficacy of DMF. ${ }^{30}$ In addition to aspirin, taking the 
medication with food and antihistamines are further recommendations given by experts. ${ }^{66}$ In addition, patients who were treated with the NYU initiation protocol had lower discontinuation rates due to flushing compared to those on Biogen protocol $(0.5 \%$ vs $3.2 \%) .{ }^{67}$

\section{Lymphopenia and liver toxicity}

The Biogen prescription label recommends obtaining a complete blood count, including ALCs prior to initiating DMF and follow up tests every 6 to 12 months. In patients with ALCs less than $0.5 \times 10^{9} / \mathrm{L}$ and persisting for $>6$ months, interruption of treatment is recommended to prevent the risk of PML. ${ }^{31}$ A retrospective study of patients who developed lymphopenia on standard maintenance dose and were subsequently switched to partial dose, ie, $240 \mathrm{mg}$ once daily or less, a reduction in degree of lymphopenia was seen following dose reduction without any decrease in efficacy, suggesting partial dose therapy as an effective approach in such patients. ${ }^{73}$

Due to increasing reports of liver injury cases in clinical practice, the Biogen prescription label recommends measuring serum aminotransferase, alkaline phosphatase (ALP) and total bilirubin levels prior to initiating DMF treatment and also while on treatment, as clinically indicated. In the event of severe liver injury, discontinuation of treatment is advised. ${ }^{31}$

\section{Conclusion}

In the past few years, there has been a considerable increase in number of approved DMTs for relapsing forms of MS, and it seems that in the coming years this list will likely grow further. With increasing choices available to the patients and physicians, individually suited selection of medication based on patient tolerability and adherence becomes more important. There is good evidence to support efficacy of DMF from two phase 3 clinical trials $\left(\right.$ DEFINE $^{33}$ and CONFIRM $^{34}$ ) and interim analysis of extension study ${ }^{41}$ (ENDORSE). DMF has also been shown to promote clinical and radiological NEDA in patients with RRMS. Furthermore, being an oral agent with relatively favorable risk versus benefit profile DMF seems to be favored by many as a first-line agent. However, its AEs especially GI dysfunction and flushing have been shown to limit patient adherence. Utilization of various useful realworld strategies, including patient education, pretreatment with aspirin, slow titration, administration of DMF with food, temporary dose reduction (if needed) and use of symptomatic therapies may serve useful in promoting patient adherence and compliance. Given the relatively recent introduction of DMF in the real world, further post-marketing surveillance is required to conclude patient's adherence and tolerability. Furthermore, health care provider will have an important role in continued surveillance and reporting of adverse events associated with DMF.

\section{Acknowledgment}

We thank Sara Vinje for the secretarial assistance.

\section{Author contributions}

Concept and design: BN and DD. Acquisition and interpretation of data: BN and DD. Drafting of the manuscript: BN and DD. Critical revision: DD. Study supervision: DD. All authors contributed to data analysis, drafting and revising the article, gave final approval of the version to be published, and agree to be accountable for all aspects of the work.

\section{Disclosure}

Dr Divyanshu Dubey reports research support from Grifols, grants from Center of Multiple Sclerosis and Autoimmune Neurology, and provided consultation to UCB, outside the submitted work. He has patent pending for KLHL11 as a marker of neurological autoimmunity and paraneoplastic disorders. The authors report no other conflicts of interest in this work.

\section{References}

1. Høglund RA, Maghazachi AA. Multiple sclerosis and the role of immune cells. World J Exp Med. 2014;4:27-37. doi:10.5493/wjem. v4.i3.27

2. Olsson T, Barcellos LF, Alfredsson L. Interactions between genetic, lifestyle and environmental risk factors for multiple sclerosis. Nat Rev Neurol. 2016;13:25. doi:10.1038/nrneurol.2016.187

3. Dubey D, Kieseier BC, Hartung HP, et al. Dimethyl fumarate in relapsing-remitting multiple sclerosis: rationale, mechanisms of action, pharmacokinetics, efficacy and safety. Expert Rev Neurother. 2015;15:339-346. doi:10.1586/14737175.2015.1025755

4. Confavreux C, Vukusic S, Moreau T, Adeleine P. Relapses and Progression of Disability in Multiple Sclerosis. $N$ Engl $J$ Med. 2000;343:1430-1438. doi:10.1056/NEJM200011163432001

5. Costello K, Halper J, Kalb R, Skutnik L, Rapp R. The use of diseasemodifying therapies in multiple sclerosis: principles and current evidence. A consensus paper by the multiple sclerosis coalition. 2015. Available from: http://www.nationalmssociety.org/getmedia/5ca284d3fc7c-4ba5-b005-ab537d495c3c/DMT_Consensus_MS_Coalition color. Accessed August 20, 2019.

6. FDA approves new oral drug to treat multiple sclerosis [online]. Available from: https://www.fda.gov/news-events/press-announce ments/fda-approves-new-oral-drug-treat-multiple-sclerosis. Accessed August 2, 2019.

7. FDA approves new oral treatment for multiple sclerosis [online]. Available from: https://www.fda.gov/news-events/press-announce ments/fda-approves-new-oral-treatment-multiple-sclerosis. Accessed August 2, 2019. 
8. Wingerchuk DM, Carter JL. Multiple sclerosis: current and emerging disease-modifying therapies and treatment strategies. Mayo Clinic Proc. 2014;89:225-240. doi:10.1016/j.mayocp.2013.11.002

9. Patti F. Optimizing the benefit of multiple sclerosis therapy: the importance of treatment adherence. Patient Prefer Adherence. 2010;4:1-9.

10. Caon C, Namey M, Meyer C, et al. Prevention and management of infusion-associated reactions in the comparison of alemtuzumab and Rebif(囚) efficacy in multiple sclerosis (CARE-MS) program. Int $J$ MS Care. 2015;17:191-198. doi:10.7224/1537-2073.2014-030

11. Agashivala N, Wu N, Abouzaid S, et al. Compliance to fingolimod and other disease modifying treatments in multiple sclerosis patients, a retrospective cohort study. BMC Neurol. 2013;13:138. doi:10.1186/ 1471-2377-13-202

12. Bergvall N, Petrilla AA, Karkare SU, et al. Persistence with and adherence to fingolimod compared with other disease-modifying therapies for the treatment of multiple sclerosis: a retrospective US claims database analysis. J Med Econ. 2014;17:696-707. doi:10.3111/13696998.2014.940422

13. Mrowietz U, Barker J, Boehncke W-H, et al. Clinical use of dimethyl fumarate in moderate-to-severe plaque-type psoriasis: a European expert consensus. J Eur Acad Dermatol Venereol. 2018;32:3-14. doi: $10.1111 /$ jdv. 15218

14. Ingwersen J, Aktas O, Hartung H-P. Advances in and algorithms for the treatment of relapsing-remitting multiple sclerosis. Neurotherapeutics. 2016;13:47-57. doi:10.1007/s13311-015-0412-4

15. Schmidt MM, Dringen R. Fumaric acid diesters deprive cultured primary astrocytes rapidly of glutathione. Neurochem Int. 2010;57:460-467. doi:10.1016/j.neuint.2010.01.006

16. Linker RA, Lee D-H, Ryan S, et al. Fumaric acid esters exert neuroprotective effects in neuroinflammation via activation of the Nrf2 antioxidant pathway. Brain. 2011;134:678-692. doi:10.1093/ brain/awq386

17. Nguyen T, Sherratt PJ, Pickett CB. Regulatory mechanisms controlling gene expression mediated by the antioxidant response element. Annu Rev Pharmacol Toxicol. 2003;43:233-260. doi:10.1146/ annurev.pharmtox.43.100901.140229

18. Wang Q, Chuikov S, Taitano S, et al. Dimethyl fumarate protects neural stem/progenitor cells and neurons from oxidative damage through Nrf2-ERK1/2 MAPK pathway. Int $J$ Mol Sci. 2015;16:13885. doi:10.3390/ijms160613885

19. Ghoreschi K, Brück J, Kellerer C, et al. Fumarates improve psoriasis and multiple sclerosis by inducing type II dendritic cells. J Exp Med. 2011;208:2291-2303. doi:10.1084/jem.20100977

20. Peng H, Guerau-de-Arellano M, Mehta VB, et al. Dimethyl fumarate inhibits dendritic cell maturation via nuclear factor $\kappa \mathrm{B}(\mathrm{NF}-\kappa \mathrm{B})$ and extracellular signal-regulated kinase 1 and 2 (ERK1/2) and mitogen stress-activated kinase 1 (MSK1) signaling. $J$ Biol Chem. 2012;287:28017-28026. doi:10.1074/jbc.M112.383380

21. Borgers M, Beyaert R, Borghmans I, et al. Dimethylfumarate is an inhibitor of cytokine-induced nuclear translocation of NF-kB1, but not rela in normal human dermal fibroblast cells. J Invest Dermatol. 2001;116:124-130. doi:10.1046/j.1523-1747.2001.00211.x

22. Lin SX, Lisi L, Dello Russo C, et al. The anti-inflammatory effects of dimethyl fumarate in astrocytes involve glutathione and haem oxygenase-1. ASN Neuro. 2011;3:e0055. doi:10.1042/AN20100033

23. Treumer F, Zhu K, Gläser R, Mrowietz U. Dimethylfumarate is a potent inducer of apoptosis in human T cells. J Invest Dermatol. 2003;121:1383-1388. doi:10.1111/j.1523-1747.2003.12605.x

24. Wu Q, Wang Q, Mao G, Dowling CA, Lundy SK, Mao-Draayer Y. Dimethyl fumarate selectively reduces memory $\mathrm{T}$ cells and shifts the balance between Th1/Th17 and Th2 in multiple sclerosis patients. J Immunol. 2017;198:3069-3080. doi:10.4049/jimmunol. 1601532
25. Gross CC, Schulte-Mecklenbeck A, Klinsing S, Posevitz-Fejfár A, Wiendl H, Klotz L. Dimethyl fumarate treatment alters circulating T helper cell subsets in multiple sclerosis. Neurology. 2016;3:e183. doi:10.1212/NXI.0000000000000183

26. Parodi B, Rossi S, Morando S, et al. Fumarates modulate microglia activation through a novel HCAR2 signaling pathway and rescue synaptic dysregulation in inflamed CNS. Acta Neuropathol. 2015;130:279-295. doi:10.1007/s00401-015-1422-3

27. Chen H, Assmann JC, Krenz A, et al. Hydroxycarboxylic acid receptor 2 mediates dimethyl fumarate's protective effect in EAE. J Clin Invest. 2014;124:2188-2192. doi:10.1172/JCI72151

28. Litjens NHR, Burggraaf J, Van Strijen E, et al. Pharmacokinetics of oral fumarates in healthy subjects. $\mathrm{Br} J$ Clin Pharmacol. 2004;58:429-432. doi:10.1111/j.1365-2125.2004.02145.x

29. Rostami-Yazdi M, Clement B, Mrowietz U. Pharmacokinetics of anti-psoriatic fumaric acid esters in psoriasis patients. Arch Dermatol Res. 2010;302:531-538. doi:10.1007/s00403-010-10 61-4

30. Sheikh SI, Nestorov I, Russell H, et al. Tolerability and pharmacokinetics of delayed-release dimethyl fumarate administered with and without aspirin in healthy volunteers. Clin Ther. 2013;35:1582-1594. e1589. doi:10.1016/j.clinthera.2013.08.009

31. Idec B. Tecfidera (dimethyl Fumarate) Prescribing Information. Cambridge (MA): Biogen Idec; 2013.

32. Edwards K, Penner N, Rogge M, Sheikh S, Zhu B. A pharmacokinetic study of delayed-release dimethyl fumarate to evaluate cerebrospinal fluid penetration in patients with secondary progressive multiple sclerosis. London: Sage Publications Lted. Mult Scler J. 2016;22:784-785.

33. Gold R, Kappos L, Arnold DL, et al. Placebo-controlled Phase 3 study of oral BG-12 for relapsing multiple sclerosis. $N$ Engl J Med. 2012;367:1098-1107. doi:10.1056/NEJMoa1114287

34. Fox RJ, Miller DH, Phillips JT, et al. Placebo-controlled Phase 3 study of oral BG-12 or glatiramer in multiple sclerosis. $N$ Engl $J$ Med. 2012;367:1087-1097. doi:10.1056/NEJMoa1206328

35. Kappos L, Giovannoni G, Gold R, et al. Time course of clinical and neuroradiological effects of delayed-release dimethyl fumarate in multiple sclerosis. Eur J Neurol. 2015;22:664-671. doi:10.1111/ ene. 12624

36. Viglietta V, Miller D, Bar-Or A, et al. Efficacy of delayed-release dimethyl fumarate in relapsing-remitting multiple sclerosis: integrated analysis of the phase 3 trials. Ann Clin Transl Neurol. 2015;2:103-118. doi:10.1002/acn3.148

37. Giovannoni G, Gold R, Kappos L, et al. Delayed-release dimethyl fumarate and disability assessed by the Multiple Sclerosis Functional Composite: integrated analysis of DEFINE and CONFIRM. Mult Scler J. 2016;2:2055217316634111. doi:10.1177/ 2055217316634111

38. Arnold DL, Gold R, Kappos L, et al. Magnetization transfer ratio in the delayed-release dimethyl fumarate DEFINE study. J Neurol. 2014;261:2429-2437. doi:10.1007/s00415-014-7504-7

39. Dupuy SL, Tauhid S, Hurwitz S, Chu R, Yousuf F, Bakshi R. The effect of dimethyl fumarate on cerebral gray matter atrophy in multiple sclerosis. Neurol Ther. 2016;5:215-229. doi:10.1007/s40120-0160054-4

40. Havrdova E, Giovannoni G, Gold R, et al. Effect of delayed-release dimethyl fumarate on no evidence of disease activity in relapsingremitting multiple sclerosis: integrated analysis of the phase III DEFINE and CONFIRM studies. Eur J Neurol. 2017;24:726-733. doi: $10.1111 /$ ene. 13272

41. Gold R, Arnold DL, Bar-Or A, et al. Long-term effects of delayed-release dimethyl fumarate in multiple sclerosis: interim analysis of ENDORSE, a randomized extension study. Mult Scler J. 2017;23:253-265. doi:10.1177/1352458516649037 
42. Berger T, Brochet B, Confalonieri P, et al. Effectiveness of delayedrelease dimethyl fumarate on clinical disease activity and patientreported outcomes in patients with relapsing-remitting multiple sclerosis in the real-world setting: a multicentre, open-label study (PROTEC). London: Sage Publications Ltd. Mult Scler J. 2016;22:298-299.

43. Everage NJ, Prada C, Liu S, et al. Safety and efficacy of delayedrelease dimethyl fumarate in multiple sclerosis patients treated in routine medical practice: interim analysis of ESTEEM (P6.333). Neurology. 2017;88:P6.333. doi:10.1212/WNL.0000000000003693

44. Smoot K, Spinelli KJ, Stuchiner T, et al. Three-year clinical outcomes of relapsing multiple sclerosis patients treated with dimethyl fumarate in a United States community health center. Mult Scler J. 2018;24:942-950. doi:10.1177/1352458517709956

45. Longbrake EE, Naismith RT, Parks BJ, Wu GF, Cross AH. Dimethyl fumarate-associated lymphopenia: risk factors and clinical significance. Mult Scler J. 2015;1:2055217315596994. doi:10.1177/ 2055217315596994

46. Weldring T, Smith SMS. Patient-reported outcomes (PROs) and patient-reported outcome measures (PROMs). Health Serv Insights. 2013;6:61-68. doi:10.4137/HSI.S11093

47. Nortvedt MW, Riise T. The use of quality of life measures in multiple sclerosis research. Mult Scler J. 2003;9:63-72. doi:10.1191/ $1352458503 \mathrm{~ms} 871 \mathrm{oa}$

48. Kita M, Fox RJ, Phillips JT, et al. Effects of BG-12 (dimethyl fumarate) on health-related quality of life in patients with relapsing-remitting multiple sclerosis: findings from the CONFIRM study. Mult Scler J. 2014;20:253-257. doi:10.1177/13524585135 07818

49. Kappos L, Gold R, Arnold DL, et al. Quality of life outcomes with BG-12 (dimethyl fumarate) in patients with relapsing-remitting multiple sclerosis: the DEFINE study. Mult Scler J. 2014;20:243-252. doi:10.1177/1352458513507817

50. Lee A, Pike J, Edwards MR, Petrillo J, Waller J, Jones E. Quantifying the benefits of dimethyl fumarate over $\beta$ interferon and glatiramer acetate therapies on work productivity outcomes in MS patients. Neurol Ther. 2017;6:79-90. doi:10.1007/s40120-016-0061-5

51. Phillips JT, Selmaj K, Gold R, et al. Clinical significance of gastrointestinal and flushing events in patients with multiple sclerosis treated with delayed-release dimethyl fumarate. Int $J$ MS Care. 2015;17:236-243. doi:10.7224/1537-2073.2014-069

52. Baharnoori M, Gonzalez CT, Chua A, et al. Predictors of hematological abnormalities in multiple sclerosis patients treated with fingolimod and dimethyl fumarate and impact of treatment switch on lymphocyte and leukocyte count. Mult Scler Relat Disord. 2018;20:51-57. doi:10.1016/j.msard.2017.12.003

53. Lehmann-Horn K, Penkert H, Grein P, et al. PML during dimethyl fumarate treatment of multiple sclerosis: how does lymphopenia matter? Neurology. 2016;87:440-441. doi:10.1212/WNL.00000000 00002900

54. Gieselbach R-J, Muller-Hansma AH, Wijburg MT, et al. Progressive multifocal leukoencephalopathy in patients treated with fumaric acid esters: a review of 19 cases. J Neurol. 2017;264:1155-1164.

55. Motte J, Kneiphof J, Straßburger-Krogias K, et al. Detection of JC virus archetype in cerebrospinal fluid in a MS patient with dimethylfumarate treatment without lymphopenia or signs of PML. J Neurol. 2018;265:1880-1882. doi:10.1007/s00415-018-8931-7

56. Muñoz MA, Kulick CG, Kortepeter CM, Levin RL, Avigan MI. Liver injury associated with dimethyl fumarate in multiple sclerosis patients. Mult Scler J. 2017;23:1947-1949. doi:10.1177/13524 58516688351
57. Tan H, Cai Q, Agarwal S, Stephenson JJ, Kamat S. Impact of adherence to disease-modifying therapies on clinical and economic outcomes among patients with multiple sclerosis. Adv Ther. 2011;28:51-61. doi:10.1007/s12325-010-0093-7

58. Gold R, Schlegel E, Elias-Hamp B, et al. Incidence and mitigation of gastrointestinal events in patients with relapsing-remitting multiple sclerosis receiving delayed-release dimethyl fumarate: a German phase IV study (TOLERATE). Ther Adv Neurol Disord. 2018;11:1756286418768775. doi:10.1177/1756286418768 775

59. Fox EJ, Vasquez A, Grainger W, et al. Gastrointestinal tolerability of delayed-release dimethyl fumarate in a multicenter, open-label study of patients with relapsing forms of multiple sclerosis (MANAGE). Int J MS Care. 2016;18:9-18. doi:10.7224/15372073.2014-101

60. Eriksson I, Cars T, Piehl F, Malmström RE, Wettermark B, von Euler M. Persistence with dimethyl fumarate in relapsing-remitting multiple sclerosis: a population-based cohort study. Eur J Clin Pharmacol. 2018;74:219-226. doi:10.1007/s00228-017-2366-4

61. Nicholas J, Boster A, Wu N, et al. Comparative effectiveness of delayed-release dimethyl fumarate versus fingolimod and teriflunomide on risk of relapse (P6.375). Neurology. 2017;88:P6.375. doi:10.1212/WNL.0000000000003693

62. Johnson KM, Zhou H, Lin F, Ko JJ, Herrera V. Real-world adherence and persistence to oral disease-modifying therapies in multiple sclerosis patients over 1 year. J Manag Care Spec Pharm. 2017;23:844-852. doi:10.18553/jmcp.2017.23.8.844

63. Vollmer B, Nair KV, Sillau SH, Corboy J, Vollmer T, Alvarez E. Comparison of fingolimod and dimethyl fumarate in the treatment of multiple sclerosis: two-year experience. Mult Scler J. 2017;3:2055217317725102. doi:10.1177/2055217317725102

64. Srivastava K, Arora A, Kataria A, Cappelleri JC, Sadosky A, Peterson AM. Impact of reducing dosing frequency on adherence to oral therapies: a literature review and meta-analysis. Patient Prefer Adherence. 2013;7:419-434. doi:10.2147/PPA. S44646

65. Utz KS, Hoog J, Wentrup A, et al. Patient preferences for diseasemodifying drugs in multiple sclerosis therapy: a choice-based conjoint analysis. Ther Adv Neurol Disord. 2014;7:263-275. doi:10.1177/1756285614555335

66. Phillips JT, Hutchinson M, Fox R, Gold R, Havrdova E. Managing flushing and gastrointestinal events associated with delayed-release dimethyl fumarate: experiences of an international panel. Mult Scler Relat Disord. 2014;3:513-519. doi:10.1016/j.msard.2014.03. 003

67. Sammarco C, Laing L, Herbert J. Strategies to reduce adverse events related to oral dimethyl fumarate. London: Sage Publications Ltd. Mult Scler J.2014;20:206.

68. Mäurer M, Voltz R, Begus-Nahrmann Y, Schmid B, Niemczyk G, Kieseier B. Adherence project with German MS-patients: can an approach of individualized patient counseling improve adherence? London: Sage Publications Ltd. Mult Scler J.2014;20:203-203.

69. Theodore Phillips J, Erwin AA, Agrella S, et al. Consensus management of gastrointestinal events associated with delayed-release dimethyl fumarate: a delphi study. Neurol Ther. 2015;4:137-146. doi:10.1007/s40120-015-0037-x

70. O’Gorman J, Russell HK, Li J, Phillips G, Kurukulasuriya NC, Viglietta V. Effect of aspirin pretreatment or slow dose titration on flushing and gastrointestinal events in healthy volunteers receiving delayed-release dimethyl fumarate. Clin Ther. 2015;37:1402-1419. e1405. doi:10.1016/j.clinthera.2015.03.028 
71. Koulinska I, Riester K, Chalkias S, Edwards MR. Effect of bismuth subsalicylate on gastrointestinal tolerability in healthy volunteers receiving oral delayed-release dimethyl fumarate: PREVENT, a randomized, multicenter, double-blind, placebo-controlled study. Clin Ther. 2018;40:2021-2030. e2021. doi:10.1016/j.clinthera.2018.10.013

72. Tornatore C, Amjad F. attenuation of dimethyl fumarate-related gastrointestinal symptoms with montelukast (P7.251). Neurology. 2014;82:P7.251. doi:10.1212/WNL.0000000000000513

73. Wong K-H, Marini E, Nguyen T, et al. Dimethyl fumarate in relapsing remitting multiple sclerosis: 24 months observations of the effects of dose reduction on lymphopenia (P6.347). Neurology. 2018;90:P6.347. doi:10.1212/WNL.0000000000005560
74. Miclea A, Leussink VI, Hartung HP, Gold R, Hoepner R. Safety and efficacy of dimethyl fumarate in multiple sclerosis: a multi-center observational study. J Neurol. 2016;263:1626-1632. doi:10.1007/ s00415-016-8175-3

75. Sejbaek T, Nybo M, Petersen T, Illes Z. Real-life persistence and tolerability with dimethyl fumarate. Mult Scler Relat Disord. 2018;24:42-46. doi:10.1016/j.msard.2018.05.007

\section{Publish your work in this journal}

Patient Preference and Adherence is an international, peer-reviewed, open access journal that focusing on the growing importance of patient preference and adherence throughout the therapeutic continuum. Patient satisfaction, acceptability, quality of life, compliance, persistence and their role in developing new therapeutic modalities and compounds to optimize clinical outcomes for existing disease states are major areas of interest for the journal. This journal has been accepted for indexing on PubMed Central. The manuscript management system is completely online and includes a very quick and fair peer-review system, which is all easy to use. Visit http:// www.dovepress.com/testimonials.php to read real quotes from published authors. 